\title{
Flapping and Flexible Wing Aerodynamics of Low Reynolds Number Flight Vehicles
}

\author{
Dragos Viieru ${ }^{1 *}$, Jian Tang ${ }^{2 \dagger}$, Yongsheng Lian ${ }^{2 \ddagger}, \mathrm{Hao} \mathrm{Liu}^{3 \S}$, and Wei Shyy ${ }^{2 * *}$ \\ ${ }^{1}$ University of Florida, Gainesville, FL, 32611, U.S.A. \\ ${ }^{2}$ University of Michigan, Ann Arbor, MI, 48109, U.S.A. \\ ${ }^{3}$ Chiba University, Chiba, Japan
}

\begin{abstract}
For flight vehicles operated at the low Reynolds number regime, such as birds, bats, insects, as well as small man-made vehicles, flapping and fixed wings are employed in various ways to generate aerodynamic forces. For flapping wings, the unsteady fluid physics, interacting with wing kinematics and shapes determine the lift generation. For fixed wings, laminarturbulent transition, three dimensional flows around low aspect ratio vehicles, and coupling between flexible wing structures and surrounding fluid flows are of major interest. In the present paper we discuss recent progress in understanding the low Reynolds number unsteady fluid dynamics associated with flapping wings, including leading-edge vortices, pitching-up rotation and wake-capturing mechanisms. For fixed wings, recent efforts in fluid-structure interaction and laminar-turbulent transition are highlighted.
\end{abstract}

\section{Introduction}

Substantial progress in the micro air vehicles (MAVs) has been reported. ${ }^{1-6}$ MAVs, with a maximum dimension of $15 \mathrm{~cm}$ and a flight speed around $10 \mathrm{~m} / \mathrm{s}$, have certain favorable scaling characteristics, including low stall velocity and low inertia, resulting in better impact survivability. However, in terms of aerodynamics, operational flight range, and maneuverability, many challenging issues are not yet well resolved. First, MAV typically operates under low Reynolds number, which, based on the nominal freestream velocity and chord length, is around $\left(10^{4}-10^{5}\right)$ conditions. Under such a low Reynolds number regime, the fluid flow is prone to separate, resulting in increases in drag and loss of efficiency. Even without flow separation, the low Reynolds number results in lower lift-to-drag ratios from $O(100)$ to $O(1)$. Second, the low aspect ratio wings employed by MAVs cause strong tip vortices, which affect a large portion of the wing, reduce the effective angle of attack, and induce three-dimensional flow structures. Third, due to its slow flight speed and low inertia, MAV is sensitive to the wind gust, making it difficult to maintain a steady flight.

Both fixed and flapping wing concepts have been explored in the MAV technologies. The fixed wing vehicle concept has been employed by, e.g., Grasmeyer and Keennon ${ }^{2}$ and Ifju et al. ${ }^{3}$ and is more mature than the flapping wing vehicle development. Figure 1(a) illustrates such a fix wing design by Ifju and coworkers. The design features a flexible wing structure. Waszak et al. ${ }^{7}$ and Lian and Shyy ${ }^{8}$ performed experimental and numerical study and showed that an MAV with such a flexible wing yields better performance than that with a rigid wing because of its shape adaptation and delay to stall. The flexible structure can be beneficial to a fixed wing as well as a flapping wing. Ho et al. ${ }^{9}$ experimentally demonstrated that a flexible wing has better lift production than a rigid wing. In the general area of fixed wing flight, Mueller and DeLaurier ${ }^{10}$ reviewed the aerodynamic performance of MAV wing under low Reynolds number conditions, and Lian et al. ${ }^{11}$ and Shyy et al. ${ }^{12}$ summarized recent efforts in numerical investigations.

\footnotetext{
* Graduate Student, Department of Mechanical and Aerospace Engineering, Student Member AIAA.

${ }^{\dagger}$ Research scientist, Department of Aerospace Engineering.

* Research scientist, Department of Aerospace Engineering, Member AIAA.

$\S$ Professor, Department of Electronics and Mechanical Engineering, Member AIAA.

${ }^{* *}$ Clarence L. "Kelly" Johnson Collegiate Professor and Chair, Department of Aerospace Engineering, Fellow AIAA.
} 


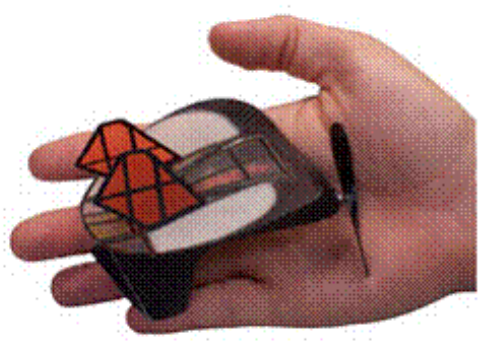

(a)

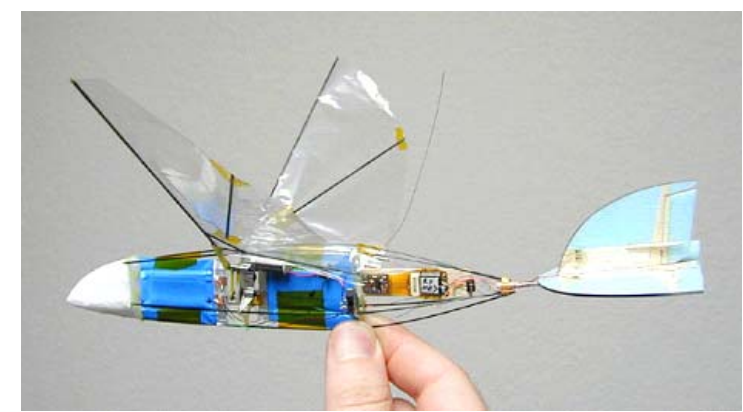

(b)

\section{Figure 1. MAV designs with fixed wing and flapping wing. (a) UF MAV (Ifju et al. ${ }^{3}$ ), (b) Microbat (Pornsin-Sirirak et al. ${ }^{1}$ ).}

The flapping wing concept, motivated by direct mimicry of birds and insects flight, attempts to produce lift and/or thrust via flapping motion. This concept has been practiced in the designs of Pornsin-Sirirak et al. ${ }^{1}$, and Jones et al. ${ }^{4}$ Figure 1(b) presents the flapping wing design of Pornsin-Sirirak and coworkers. Regarding natural flyers, Figures 2(a) and (b) illustrate forward and backward wing movements of a hummingbird, forming a figure-eight pattern, as schematically illustrated in Fig. 2 (c) and (d). In addition to the flapping wings, the tail exhibits substantial control authority to position, turn, and adjust the flight trajectory.

In this paper, we review the recent progress in both flapping and fixed wing aerodynamics motivated by our interests in the low Reynolds number air vehicles. For fixed wing, our focus is on the low Reynolds number phenomena. For flapping wing, we discuss the four lift generation mechanisms proposed to explain how insects and birds utilize unsteadiness to produce lift. For fixed wing, issues associated with the fluid-structure interactions, the laminar-to-turbulent transition, and the vortical flow structures around a low aspect wing will be highlighted.

The available literature in the relevant areas is vast, and can't be covered in the present paper. We mention a few additional aspects to promote further exchange of ideas. The flapping wings are employed to produce required lift as well as thrust. For the propulsion aspect of the flapping wing, we refer to Heathcote et al. ${ }^{13}$ and Tuncer and Platzer. ${ }^{14}$ The vortical flow structures associated with high angles-of-attack and short aspect ratio wings have been extensively investigated, as reviewed by Gursul. ${ }^{15,16}$ In the aerodynamics community, fluid flows around pitching airfoils have been reported by various researchers, e.g., Refs. ${ }^{17-23}$ These studies typically focus on higher Reynolds number regimes. Nevertheless, there is a need to draw information from different topical areas to foster better information exchange.

\section{High Lift Mechanism of Flapping Wings}

Following Ellington's research ${ }^{24}$ showing that quasi-steady analysis substantially under-predicts the aerodynamic force needed to sustain the insect weight, recent flapping wing research has focused on the understanding of unsteady aerodynamic mechanisms resulting from the wing movement. In flapping flight one measure of the degree of unsteadiness is the reduced frequency defined as follows:

$$
k=\frac{\omega c}{2 U},
$$

where $\omega$ is the wing angular velocity, $c$ is the root chord length, and $U$ is the magnitude of the forward speed.

Figure 3 shows the trend between the mass of the various birds and insects and the corresponding reduced frequency. The data are based on those reported by Azuma ${ }^{25}$ and Pennycuick, ${ }^{26}$ aided by the cruising velocity estimate documented by Tennekes. ${ }^{27}$ Overall, the reduced frequency decreases as the vehicle size and mass grows, indicating that small flyers employ more unsteadiness in their flight than large flyers. Although this figure does not explain how the unsteadiness is used, it does disclose that unsteadiness plays a critical role in small flyers' movement. 


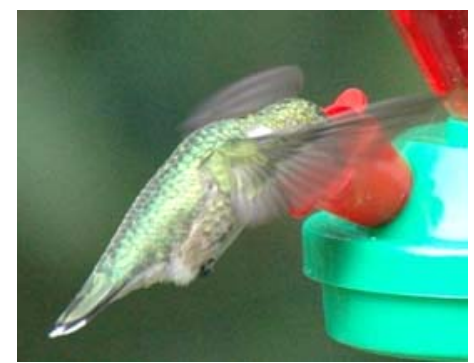

(a)

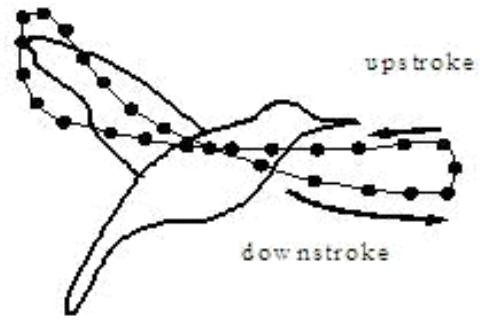

(c)

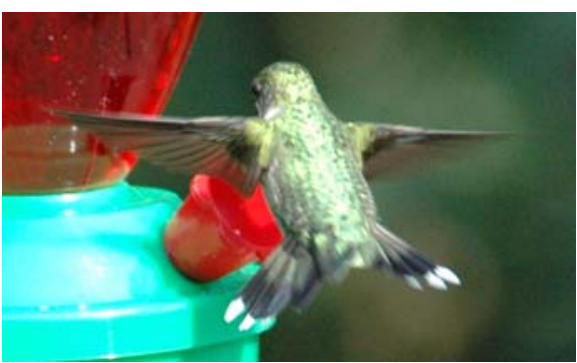

(b)

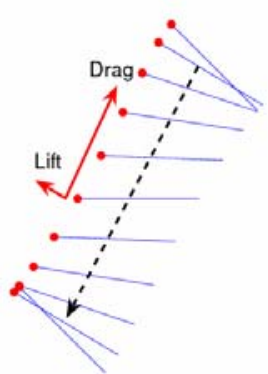

(d)

Figure 2. Illustration of biological flapping wing patterns: (a) forward stroke, and (b) backward stroke (picture taken by Wei Shyy), (c) the figure eight pattern (adopted from Azuma ${ }^{25}$ ), and (d) the trajectory and angle-of-attack of wing during a single period, where the downstroke and upstroke phases are indicated by red arrows. The circles indicate the leading edge.

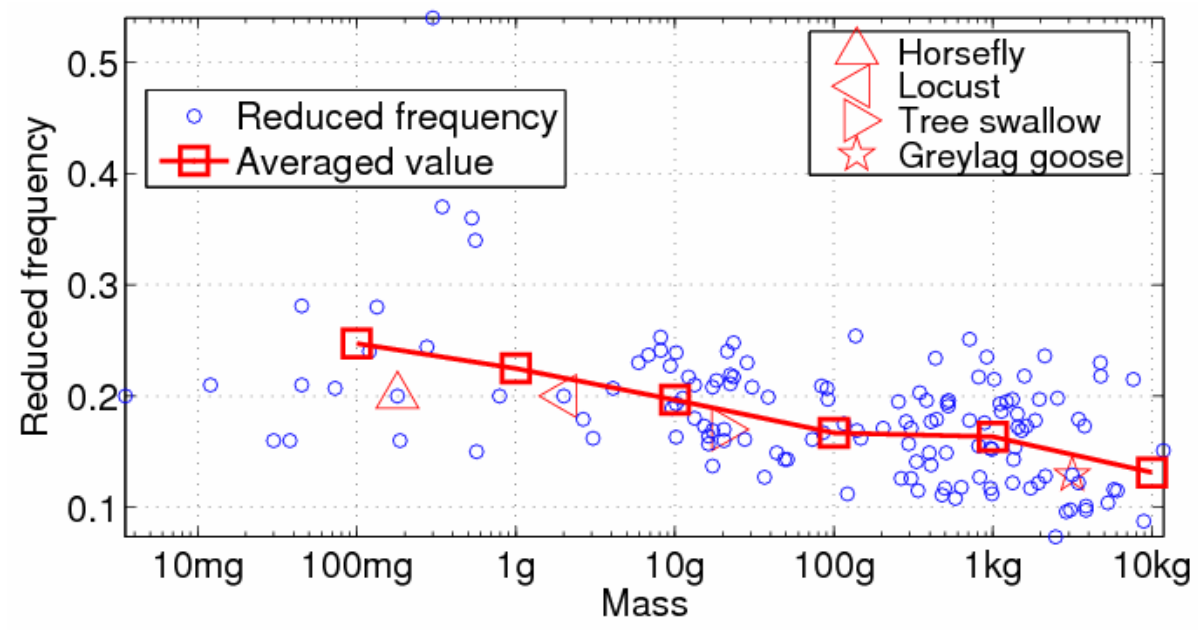

Figure 3. Mass versus reduced frequency for birds and insects.

Alternative theories have been proposed to help explain the physical responsible for lift generation, including delayed stall phenomenon associated with the unsteady wing movement, ${ }^{28,29}$ upper wing leading-edge vortices, ${ }^{24,30}$ fast pitching-up, ${ }^{31,32}$ and wake-capturing ${ }^{29}$ (also termed fast acceleration ${ }^{31,32}$ ). In the following, we will discuss four major mechanisms associated with the flapping wing aerodynamics, namely,
A. Weis-Fogh's clap-and-fling.
B. Leading-edge vortices.
C. Pitching-up rotation.
D. Wake-capturing. 
While alternative scenarios have offered improved insight into the lift generation mechanisms, further probing is essential before we can reach concrete conclusions regarding the physics, and the necessary guidelines regarding vehicle design concepts. In the following, we highlight the research related to these four lift generation mechanisms. Representative studies have been summarized in the tables in Appendix.

\section{A. Weis-Fogh's clap-and-fling mechanism}

Weis-Fogh, ${ }^{33}$ when studying the flight of the small wasp (Encarsia Formosa), found that at the end of upstroke and at the beginning of the downstroke, the two wings clapped together (clap) and then peeled apart (fling). The termed "clap-and-fling" causes large circulation and generates considerably large lift on the wing. A schematic shown in Fig. 4 demonstrates this mechanism. Lighthill ${ }^{34}$ modeled the fling phase and showed that a circulation proportional to the angular velocity of the fling was generated. Maxworthy, ${ }^{35}$ by flow visualization experiment on a pair of wings, discovered that during the fling process, a leading edge vortex was generated on each wing and its circulation was substantially larger than that calculated by Lighthill. ${ }^{34}$ Later, Ellington used "clap-and-fling" to explain the flapping behavior of birds and bats during take-off and landing.

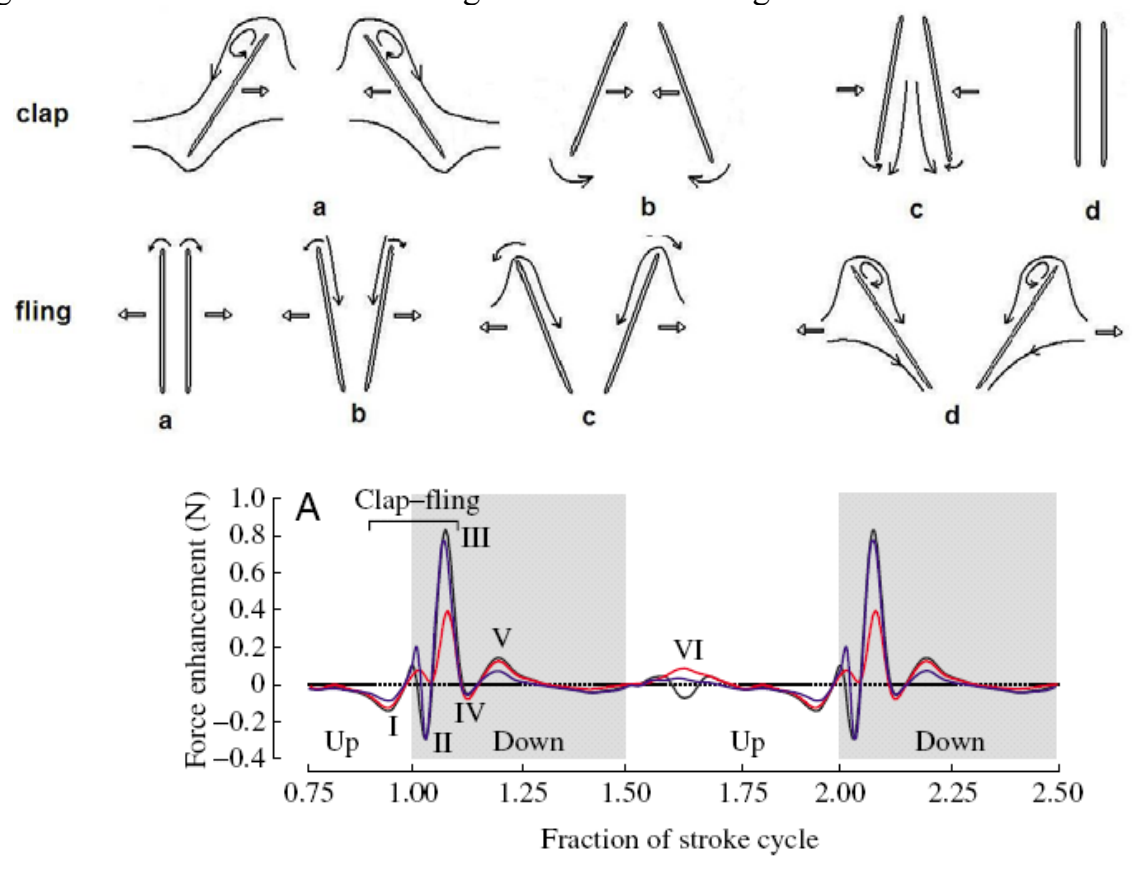

Figure 4. Clap-and-fling mechanism drawn based on Weis-Fogh ${ }^{33}$ and experimental demonstration ${ }^{36}$ of the force enhancement based on robotic wings. In lower figure, force differences between single wing and wings performing clap-and-fling in lift production are shown in red and differences in drag are plotted in blue. The time traces demonstrate that dorsal wing interaction due to clap-and-fling may augment but also diminish force and lift production throughout the entire stroke cycle. Roman numbers (I-VI) label the main force peaks found in the data traces. (The lower figure is reproduced from Ref. 36 with permission from F. $O$. Lehman).

Lehmann ${ }^{37}$ and Lehmann et al..$^{36}$ experimentally investigated force enhancement based on a dynamically scaled mechanical model of the small fruit fly Drosophila melanogaster. Their study is highlighted in the lower portion of Fig. 4. Apparently, the contra-lateral wing interactions during the stroke reversal period of the clap-and-fling process can substantially affect the aerodynamics. Specifically, their results suggested that lift enhancement during clap-andfling can be up to $17 \%$ if an angular separation between the two wings is about $10-12^{\circ}$.

\section{B. Leading-edge vortices}

Delayed dynamic stall associated with unsteady fluid flow can also help explain the extra lift of insect wings. Dickinson and Götz ${ }^{28}$ measured the aerodynamic forces of an airfoil started rapidly at high angles of attack in the Reynolds number range of fruit fly wing (Reynolds numbers $=75-225$ ). They showed that lift was enhanced by the presence of the leading-edge vortices (LEV). The leading-edge vortices generate a lower pressure area which results in a large suction on the upper surface. Two-dimensional study showed that the lift enhancement could sustain for 3 
or 4 chord lengths of travel before vortex breakdown occurs. On the other hand, experimental work by Ellington et al. ${ }^{30}$ showed that the leading-edge vortices did not breakdown. They speculated that the vortex stability is maintained by the removal of vorticity through a spanwise axial flow along the vortex core (Fig. 5). Liu and Kawachi $^{38}$ conducted unsteady Navier-Stokes simulations of the flow around a hawkmoth's wing. Their computations confirmed the leading-edge vortex and the spanwise flow discovered by van den Berg and Ellington ${ }^{39}$ and Ellington et al. ${ }^{30}$ In another effort, Sane and Dickinson, ${ }^{40}$ by placing a fence on the upper surface of the robotic model wing, showed that the LEV still exists on the wing. Based on this observation, they suggested that the spanwise flow should not be responsible for the LEV on the upper wing surface.

As the flyers' sizes and their flight Reynolds number vary, the relative importance among viscous, convective, and pressure terms changes accordingly, which exerts substantial impact on the structures of the leading-edge vortices. Figure 6 highlights the streamlines at three Reynolds numbers. Figure 6(a) corresponds to a hawkmoth with a Reynolds number of 6,000, Figure 6(b) corresponds to a fruit fly with a Reynolds number of 120, and Figure 6(c) corresponds to a thrips with a Reynolds number of 10 . At the Reynolds number of 6,000 , as observed in previous studies, ${ }^{30,38}$ an intense, conical leading-edge vortex core is observed on the paired wings with a sustained spanwise flow at the vortex core, breaking down at approximately three-quarters of

(a)
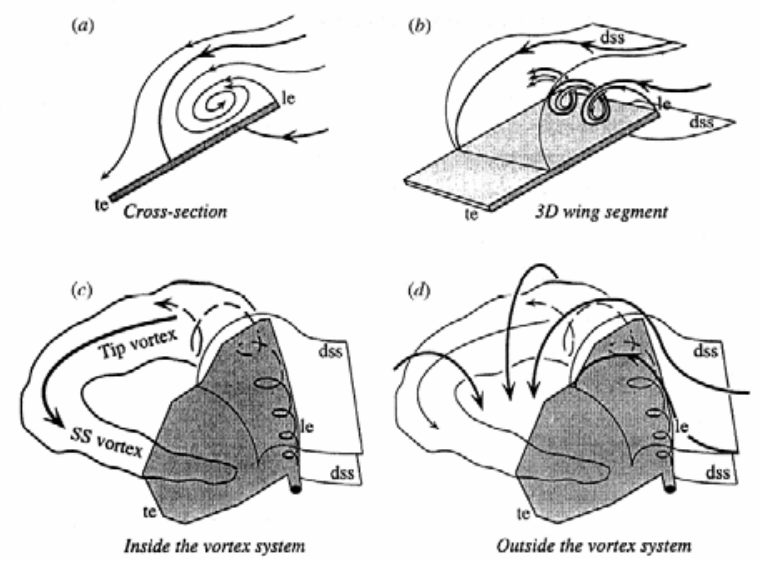

Figure 5. Spatial flow structure of leading-edge vortices. (Reproduced from Ref. 39 with permission from C. P. Ellington. $l e=$ leading edge, $t e=$ trailing edge, $d s s=$ dividing stream surface, $S S$ vortex $=$ combined starting/stopping vortex. )

the span towards the tip. At the Reynolds number of 120 (Fig. 6(b)), the vortex no longer breaks down and is found to be connected to the tip vortex. The spanwise flow at the vortex core becomes weaker as the Reynolds number is lowered, which is in qualitative agreement with the findings of Birch and Dickinson. ${ }^{41}$ Further reducing the Reynolds number to 10, a vortex ring, which connects the leading-edge vortex, the tip vortex and the trailing vortex, is observed (Fig. 6(c)).

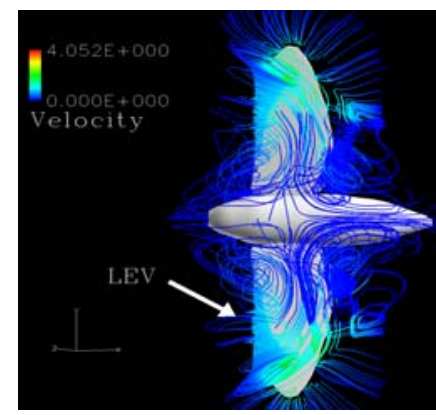

(a)

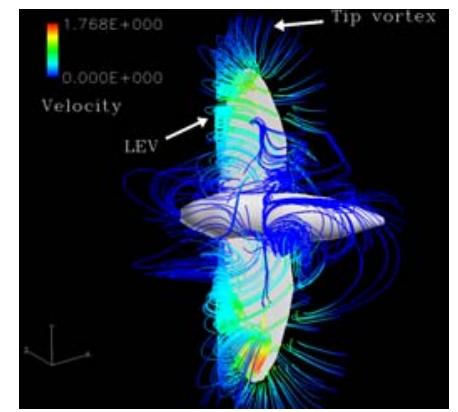

(b)

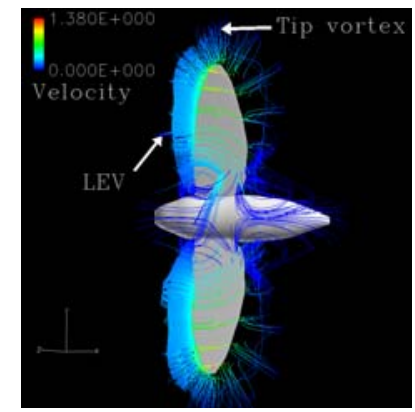

(c)

Figure 6. Numerical results of leading edge vertical structures at different Reynolds numbers. (a) Reynolds number $=6,000$, (b) Reynolds number $=120$, (c) Reynolds number $=10$.

Their flow structure shows more of a cylindrical than a conical form. Inspecting the momentum equation, one can see that the pressure-gradient, the centrifugal force, and the Coriolis force together are responsible for the leading-edge vortex stability. Their roles at different Reynolds numbers should be further studied to help shed light on these findings. 


\section{Pitching-up rotation}

Dickinson et al. $^{29}$ used a dynamically scaled robotic model to simulate the hovering flight of the fruit fly, drosophila. They found two aerodynamic force peaks in each flapping stroke. These peaks were confirmed by numerical simulations of Sun and Tang ${ }^{31,32}$ and Ramamurti and Sandberg. ${ }^{42}$ As shown in Fig. 7, the first peak, termed "rotational force" by Sane and Dickinson, ${ }^{40}$ appears near the end of each stroke. In advanced rotation, the wing flips before reversing its translational direction as illustrated in Fig. 8(f) and (g), the leading edge rotates backward relative to the translation, and this mechanism, akin to the Magnus effect, produces lift. This explanation of Dickinson et al., ${ }^{29}$ shown in Fig. 9, is intuitive; one can't extend the same interpretation from a cylinder to a thin airfoil. ${ }^{43}$ Based on their computational analysis, Sun and Tang ${ }^{31}$ suggested that the first peak is due to fast vorticity increase when the wing experiences fast pitching-up rotation (termed "pitching-up mechanism"). The pitching-up rotation and the associated vorticity increase are plotted in Fig. 8(f) and $(\mathrm{g})$. Walker ${ }^{44}$ applied the unsteady blade element method with empirically derived force coefficients to interpret this force peak. He

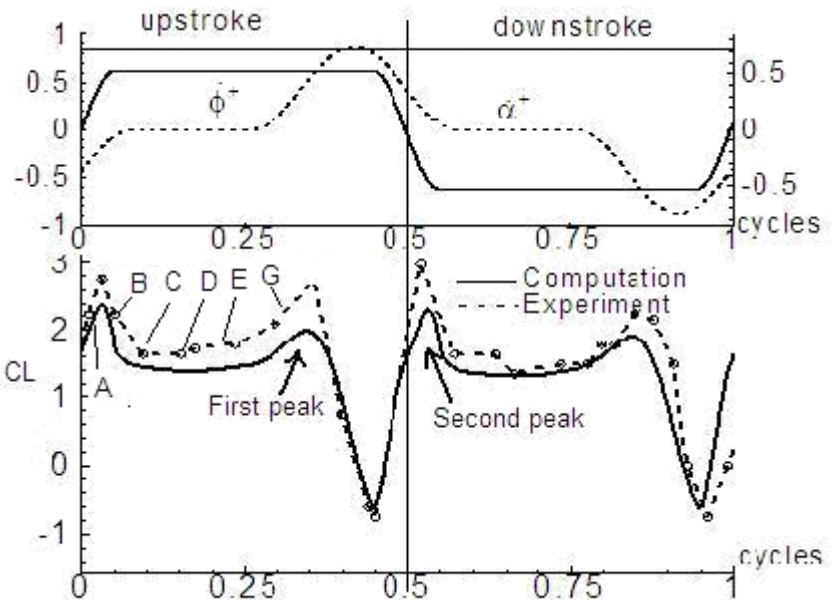

Figure 7. Experimental and numerical results show the two lift peaks in each stroke (Sun and Tang ${ }^{31}$ ).

suggested that "the rotational lift is not a fundamental aerodynamic mechanism, but simply a kinematic mechanism that augments incident-angle-dependent circulation and the resulting circulatory-and-attached-vortex force". Based on Walker's investigation, Sane and Dickinson ${ }^{40}$ attributed the first force peak to the additional circulation generated to reestablish the Kutta condition during the rotation. Overall, the findings reported by Sun and Tang ${ }^{31}$ and Sane and Dickinson $^{40}$ are in general agreement.
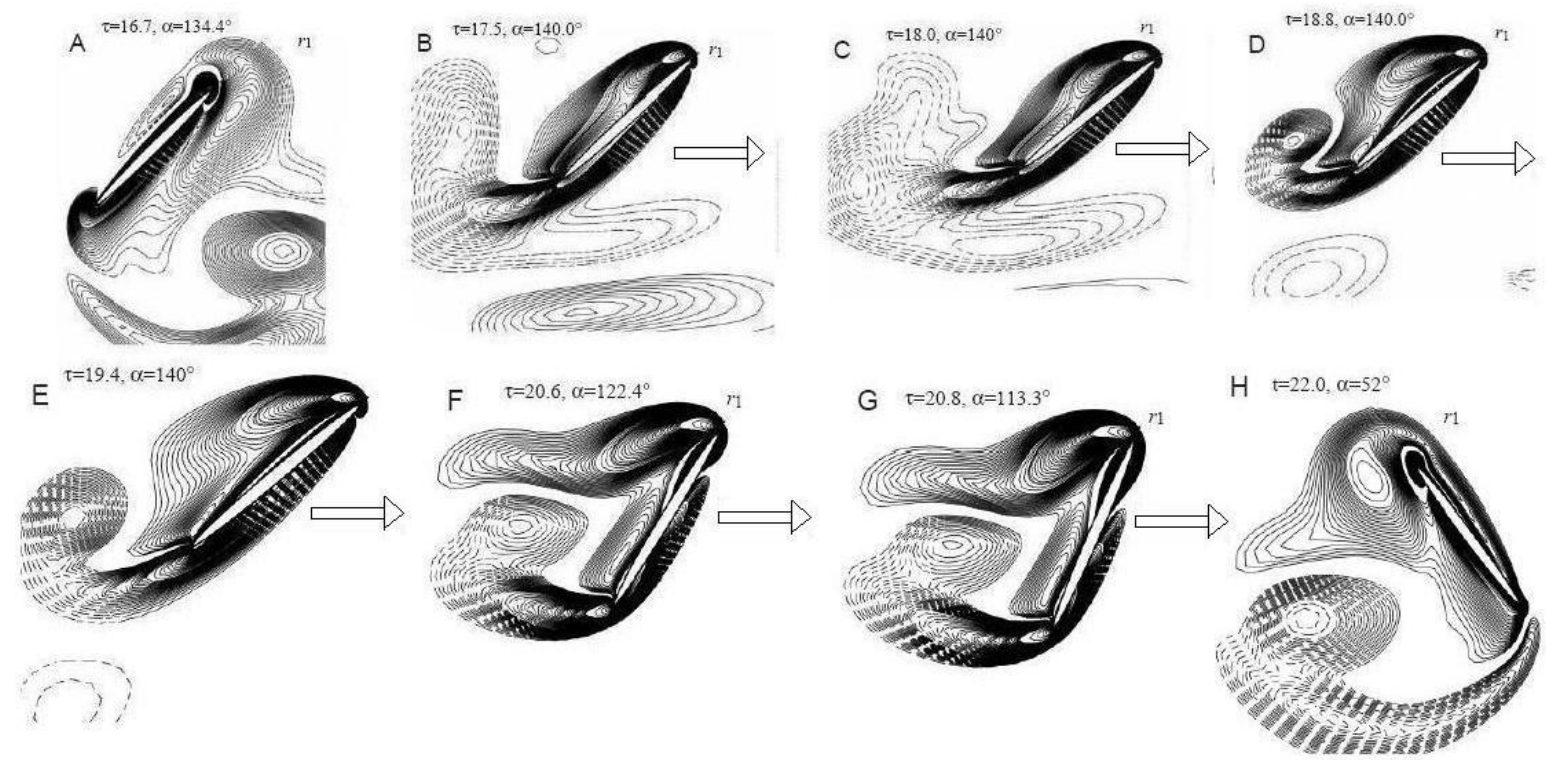

Figure 8. Vorticity distribution at $75 \%$ wingspan during rapid pitching-up rotation (Sun and Tang ${ }^{31}$ ). 


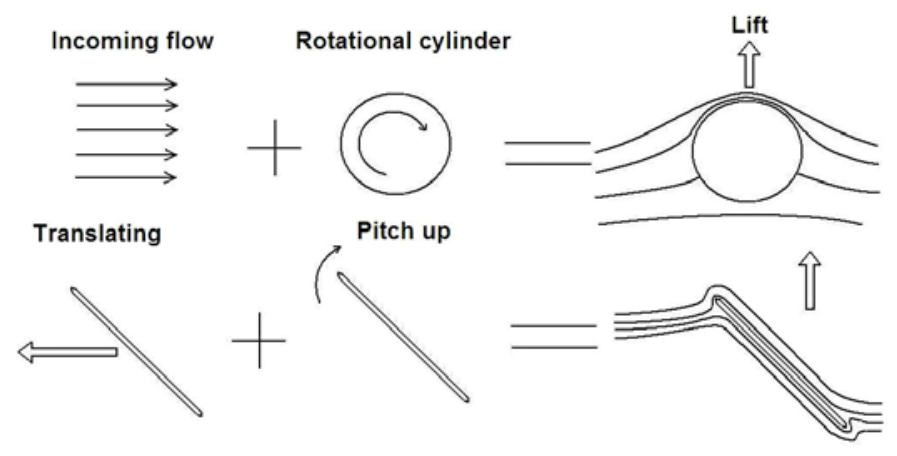

Figure 9. The Magus effect generates enhanced lift due to cylinder rotation. However, it is inappropriate to apply the same interpretation to a thin airfoil due to very different fluid physics in both geometries.

\section{Wake-capturing}

The second peak, termed wake-capturing, is related to the wing wake interaction. It is generated at the beginning of each stroke of hovering flight when the wings reverse the direction of moving while rotating about the spanwise direction. This movement is shown in Fig. 8(a) and (b). Wings meet the wake created during the previous stroke after reversing their direction, which increases the effective flow velocity and generates the second force peak. The wake-capturing mechanism is illustrated in Fig. 10. In the two-dimensional numerical simulation of Wang et al., ${ }^{45}$ the wake-capturing mechanism was proven to increase the lift peak at the beginning of the stroke.

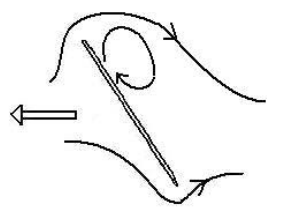

a

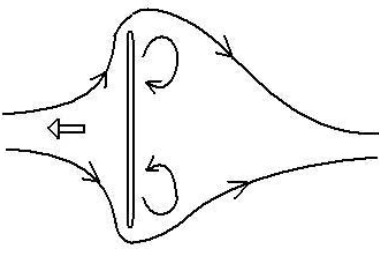

b

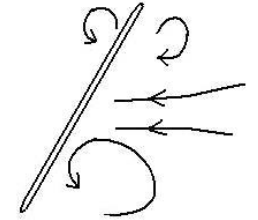

C

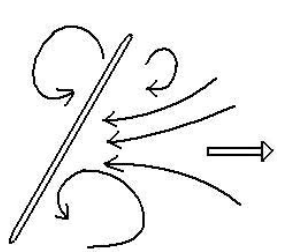

d

Figure 10. Momentum transfer in wake-capturing interaction. (a) wing is steadily translating, (b) trailing edge vortex are generated as the wing rotates around a spanwise axis, (c) leading edge vortex generated when wing rotates at the end of the stroke, (d) wing reverses flapping direction and encounters the induced velocity field by the previous shed pair of vortices and fluid momentum is transferred to the wing to generate a force. (Drawings based on Dickinson et al. ${ }^{29}$ )

The wake-capturing mechanism is also observed recently in our investigation of the hovering flight. We studied the hovering of a two-dimensional elliptic airfoil with $15 \%$ thickness moving according to the following equations:

$$
\begin{gathered}
h(t)=h_{a} \sin (2 k t+\phi) \\
\alpha(t)=\beta+\alpha_{a} \sin (2 k t),
\end{gathered}
$$

where $h_{a}$ is the plunging amplitude, $\beta$ is the initial pitching angle, $\alpha_{a}$ is the pitching amplitude, $\phi$ is the phase difference between pitching and plunging motion, and $k$ is the reduced frequency.

Two different hovering modes have been studied: Mode 1 is based on the work of Liu and Kawachi, ${ }^{38}$ and Mode 2 is similar to the work of Wang et al. ${ }^{45}$ Mode 1 has the parameter of $\alpha_{a}=42^{\circ}, h_{a}=1.0, \beta=0^{\circ}$, and $\phi=\pi / 2$;

Mode 2 has the same parameters as Mode 1, except that $\beta=90^{\circ}$. Both modes have the reduced frequency $k$ of 0.5 and Reynolds number, based on the maximum velocity, of 1,700 . For both hovering modes, the rotation is considered to be symmetrical since the rotation starts in the first half-stroke and ends at the beginning of the second 
half-stroke. The schematic of both hovering Mode 1 and Mode 2 is presented in Fig. 11. The laminar, incompressible Navier-Stokes equations in an inertial frame of reference are solved using a pressure-based solver. ${ }^{46}$
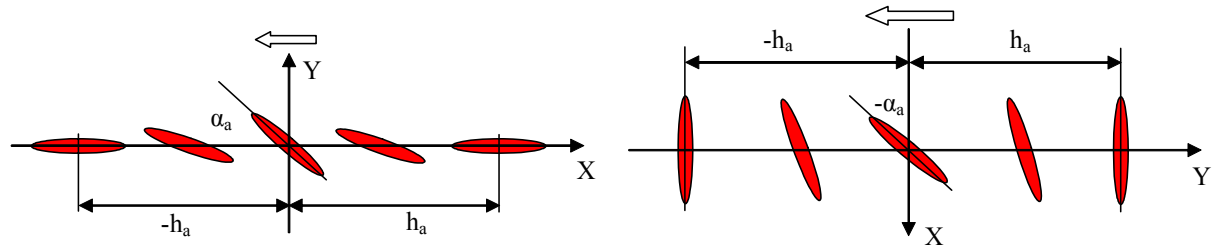

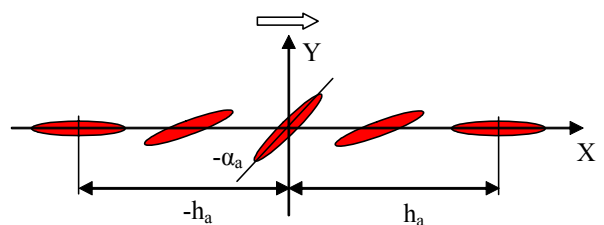

(a)

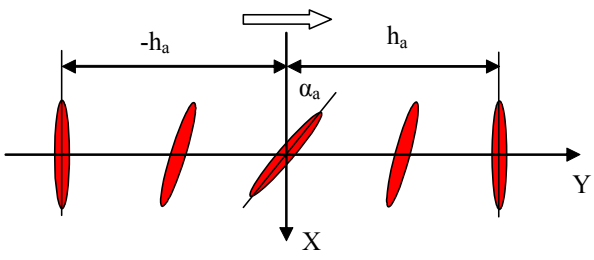

(b)

Figure 11. Schematics of hovering modes. (a) hovering Mode 1, (b) hovering Mode 2.

\section{Hovering Mode 1}

The time history of the lift coefficient during a complete flapping period, shown in Fig. 12, indicates that different mechanisms are involved in generating the lift. Figure 12(a) shows almost identical lift variation during the two half-strokes. The maximum lift peak is achieved between time $/ T=0.25$ to 0.30 for the forward stroke and for time $/ T=0.7$ to 0.8 for the backward stroke (Fig. 12(a)), where $T$ is the flapping period. The lift increases during pitching-up and decreases after the pitch angle is reduced and the leading edge vortex is shed. Figure 13(a) indicates that a pair of co-rotating vortices is shed during each of the half stroke, resulting in no significant difference in lift between the two half strokes. It seems that the wake-capturing mechanism does not play an important role in hovering Mode 1 and the lift peaks are primarily generated by rapid pitching-up mechanism.

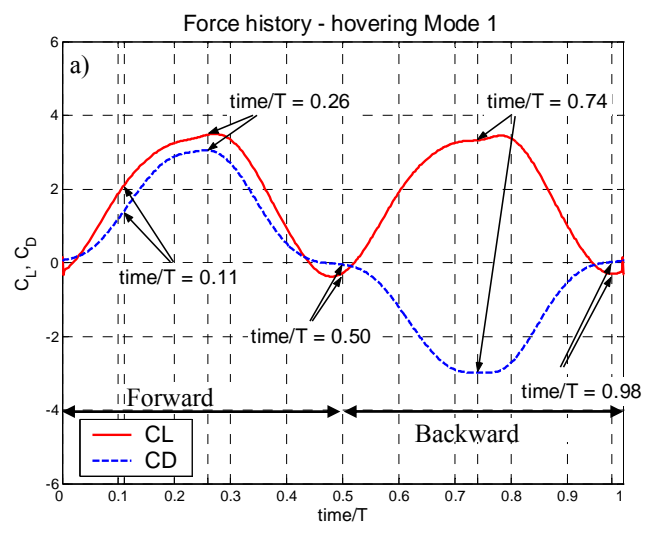

(a)

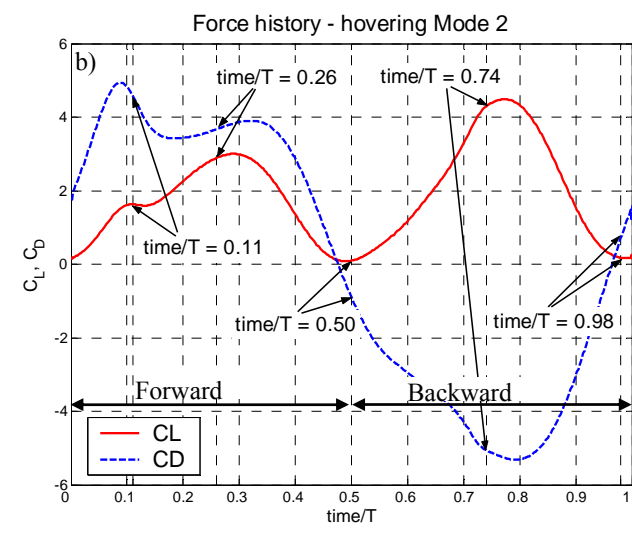

(b)

Figure 12. Force history for one period. (a) hovering Mode 1, (b) hovering Mode 2. The times when the flow snapshots were taken are indicated. Here $T$ is the flapping period. The Reynolds number is 1,700 and the reduced frequency $k=0.5$.

\section{Hovering Mode 2}

For hovering Mode 2, as shown in Fig. 12(b), the lift peak generated in the second half stroke is about $50 \%$ larger than that in the first half stroke. The vorticity contour plots in Fig. 13(b) show that during each of the half stroke, a pair of counter-rotating vortices is shed. In the second half of the stroke the wing encounters the existing pair of counter-rotating vortices and momentum from the wake is transferred to the wing. These findings are consistent with those reported by Wang et al. $^{45}$ This phenomenon supports a wake-capturing mechanism, as evidenced by the increased peak in the lift coefficient during the second half stroke. Also, one notices that the lift 
generated by hovering Mode 1 in the first half stroke has almost the same maximum value as hovering Mode 2, while in the second half-stoke the force generated by hovering Mode 2 is $20 \%$ larger. Even though the lift peaks have different magnitudes between Mode 1 and 2, the average lift coefficients generated in a stroke for both hovering modes are almost the same.

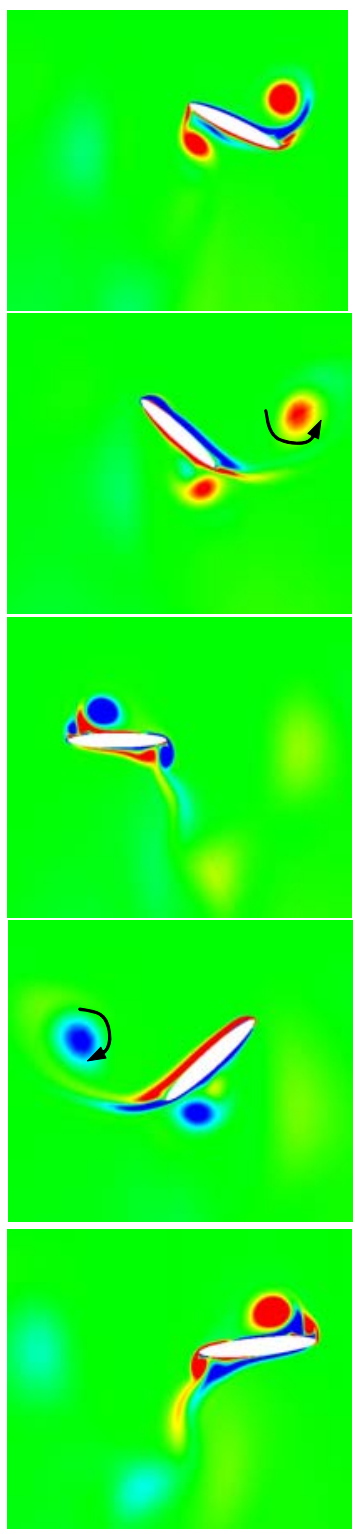

(a)

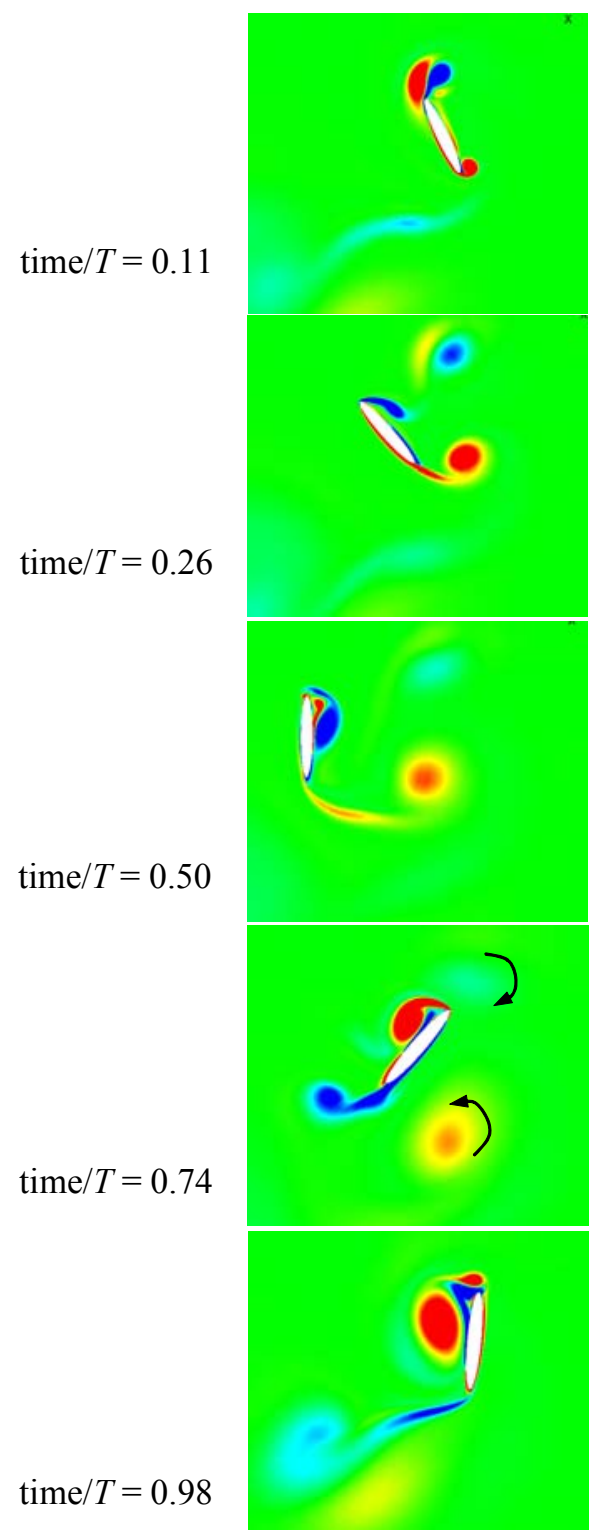

(b)

Figure 13. Vorticity contours snapshots for two hovering modes during one period. (a) hovering Mode 1; (b) hovering Mode 2. Red =counter-clockwise rotating vortices, Blue=clockwise rotating vortices. Here $T$ is the flapping period. The Reynolds number is 1,700 and the reduced frequency $k=0.5$.

We further compare our numerical results with experimental results of Wang et al. ${ }^{45}$ It should be emphasized that our Mode 2 results are based on the Reynolds number of 1,700, and the maximal hovering magnitude of one chord length. On the other hand, the experimental setup was for the Reynolds number of 75 and hovering magnitude of 2.8 chord length. Given all these differences, as shown in Fig. 14, the computational results still share substantial similarity in flow patterns: both computational and experimental results show a stronger leading edge vortex during the second half of the stroke than that presented during the first half-stroke, indicating that a wake-capturing mechanism seems to be responsible for the differences between the LEV strength during the two half-strokes. 
However, because the flapping amplitude is much smaller in the numerical simulation, the vortex pair does not have enough time to be shed, resulting in a stronger vortex pair than the experimental one.
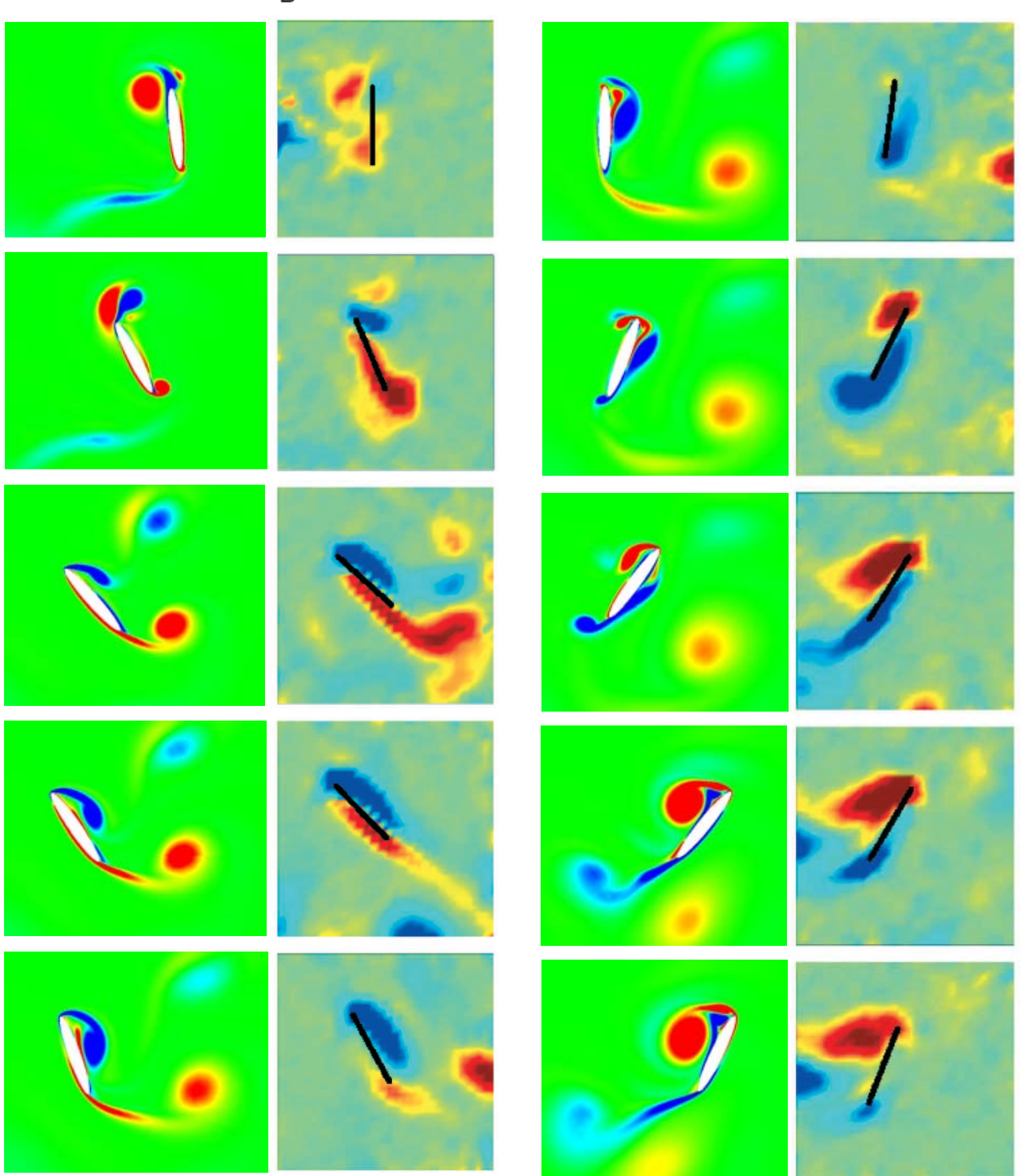

(a)

(b)
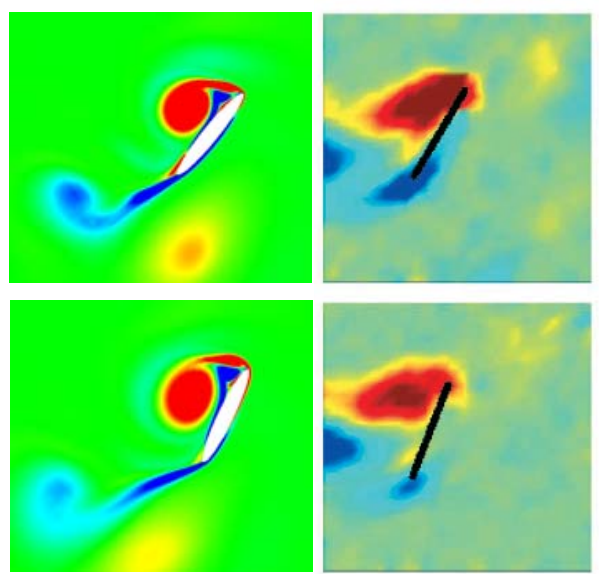

(c)

(d)

Figure 14. Vorticity plot. Red $=$ counter-clockwise rotating vortices, Blue $=$ clockwise rotating vortices. For all cases, the stroke reversal is symmetric. (a), (c) computed vorticity; (b), (d) DPIV data in a 2D slice at 0.65R. The frames are measured during the fourth stroke. The color scales for computed and measured vorticity plots do not correspond to the same contour values and should be viewed as a qualitative comparison. Also the time at which the snapshots were taken is not identical between the experiments and computations. Experimental vorticity contours adopted from Wang et al. ${ }^{45}$

Tang ${ }^{47}$ explored the effects of kinematic mode and Reynolds number on high-lift mechanisms. He found that if the translational velocity changes mildly during the stroke, as shown in Fig. 14(a), the two lift peaks would diminish or even disappear. However, the delayed stall mechanism still exists and the energy consumption is lower in this case. Given the fact that most insects change their translational velocity mildly during the flight, Tang ${ }^{47}$ suggested that the LEV caused delayed stall mechanism is more popular than other mechanisms. As illustrated in Fig. 14(b), Tang further demonstrated that the trend of force variation in each stroke did not seem sensitive to the Reynolds number, indicating that insects might apply the similar high-lift mechanisms. Along this path work has been 
extended by Wu and Sun, ${ }^{48}$ Sun and $\mathrm{Du},{ }^{49}$ and Sun and Lan. ${ }^{50}$ Of course, more efforts are required to ensure that the numerical dissipation didn't effectively reduce the actual Reynolds number being simulated.

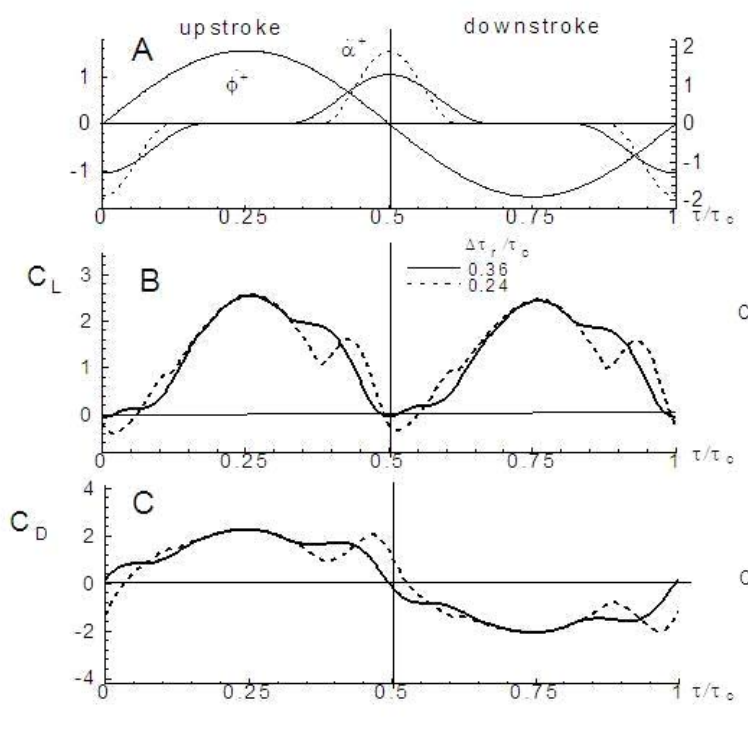

(a)
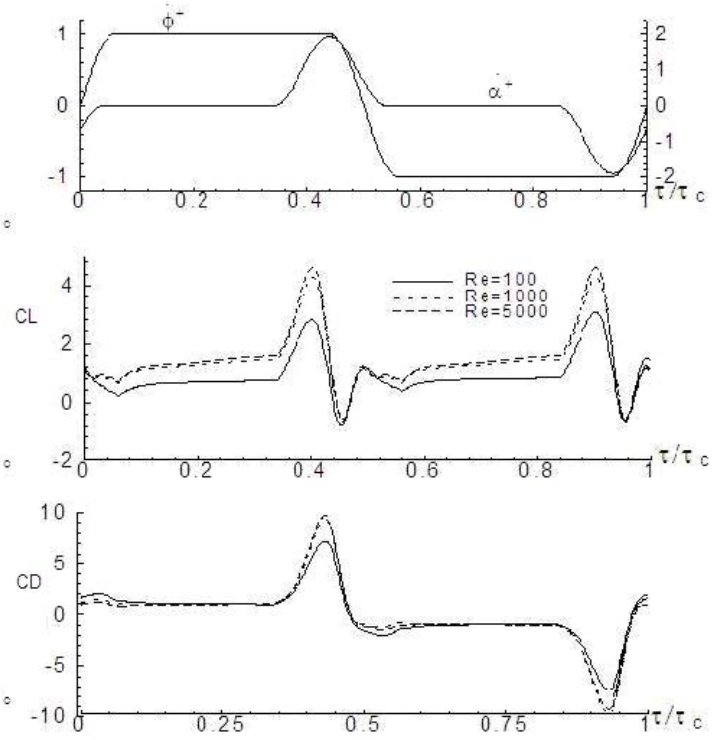

(b)

\section{Figure 15. Effects of flapping velocity and Reynolds number on aerodynamic forces (Tang ${ }^{47}$ ).}

Birch and Dickinson ${ }^{51}$ compared the aerodynamic force of the first stroke (without the wake effect) with that of the fourth stroke (in the presence of the wake of the prior strokes), and they showed that the force peak in the beginning of the stroke was partially contributed by the interaction between the wing and the wake left by the previous strokes. Sun and $\mathrm{Du}^{49}$ computed the aerodynamic forces of eight species of insect in free hovering flight. They assumed that the translational velocity of the wings varied with time as the simple harmonic function and the wing rotation was symmetrical based on the flight data. The computed mean lift balanced the insect weight. They suggested that most insects produce high lift using the delayed-stall (or LEV) mechanism in flight. Further investigation is needed to clarify the mechanism responsible for the first lift peak. However, the wake-capturing mechanism can help explain the insect's ability to extract energy from their own wake, recovering energy from air lost during the previous stroke and, therefore improving the overall force production efficiency. Overall, the two force peaks attribute a large portion of the total lift.

\section{Fixed Wing Aerodynamics}

The current MAVs typically employ fixed wings. On one hand, fixed wing is simple in concept and easy to be implemented. On the other hand, fixed wing, as a miniature of large airplane wing, deteriorates its performance because of the presence of separation bubble as its operating Reynolds number drops to the range of $10^{4}$ to $10^{5}$. On that reason, the fixed wing research focus is on the low Reynolds number aerodynamics, including the leading edge separation bubble and the thereafter incurred laminar to turbulence transition. As previously discussed, fixed wing can use flexible material as its lifting surface. This concept is practiced in the MAV design shown in Fig. 1(a). One advantage for the flexible wing is that it can facilitate passive shape adaptation, which results in delayed stall. It has been experimentally shown that under modest angles of attack, both rigid and membrane wings demonstrate similar lift characteristics with the stiffer wings having slightly higher lift coefficient. ${ }^{7}$ However, it is clear that the membrane wings stall at higher angles of attack than the rigid wing. This aspect is a key element in enhancing the stability and agility of MAVs. For example, typical rigid wings have stall angles between 12 and 15 degrees, while the stall angles of the flexible wings are between 30 and 45 degrees. ${ }^{7}$ With either design, MAVs operate in a low Reynolds number regime where the flow is sensitive to small changes in the free stream, which can either promote or inhabit separation and the transition to turbulence. This sensitivity is highlighted in Table 1, which compares the measurements among three different units on a low Reynolds number airfoil SD7003. ${ }^{52}$ With slight change in the freestream turbulence level, the transition position varies as much as $20 \%$ along the chord. 
Table 1. Measured and computed SD7003 LSB properties at $\mathrm{Re}=60,000$ and $4^{\circ}$ angle of attack. IAR $=$ the Institute for Aerospace Research, TU-BS = the Technical University of Braunschweig, and AFRL = Air Force Research Lab. Here $c$ is the airfoil chord length.

\begin{tabular}{cccccc}
\hline Data Set & $\begin{array}{c}\text { Freestream } \\
\text { turbulence, Tu[\%] }\end{array}$ & $\begin{array}{c}\text { Separation } \\
x_{s} / c\end{array}$ & $\begin{array}{c}\text { Transition } \\
x_{t} / c\end{array}$ & $\begin{array}{c}\text { Reattachment } \\
x_{r} / c\end{array}$ & $\begin{array}{c}\text { Max Bubble } \\
\text { Height }, h_{b} / c\end{array}$ \\
\hline IAR & 0.0 & 0.33 & 0.57 & 0.63 & 0.027 \\
TU-BS & 0.1 & 0.30 & 0.53 & 0.62 & 0.028 \\
AFRL & $\sim 0.1$ & 0.18 & 0.47 & 0.58 & 0.029 \\
\hline
\end{tabular}

If the incoming flow is laminar, the boundary layer often reaches separation due to the adverse pressure gradient, and the separated flow quickly undergoes transition to turbulence. Depending on the local Reynolds number, pressure gradient, surface roughness, and freestream turbulence intensity, the turbulent free shear layer can entrain enough high momentum fluid through diffusion to reattach to the surface as a turbulent boundary layer and form a laminar separation bubble (LSB). Understanding the onset of separation and subsequent reattachment has considerable practical significance because they are related to the upper limit of efficiency of the lifting bodies.

To further illustrate the salient features of LSB, we present numerical simulations based on the experiment of $\mathrm{Ol}$ et al. ${ }^{52}$ The SD7003 airfoil is used as the geometry to experimentally study LSB and transition. The primary reason to choose this geometry is that the long, stable LSB exhibits over a broad range of angles of attack at Reynolds numbers below 100,000. A detailed comparison of instruments and experimental results can be found in the work of Ol et al. ${ }^{52}$

Our focus is on the angle of attack of $4^{\circ}$, for which most of the measurements are done. Figure 16 compares the numerical results and the experimental measurement. The numerical simulation is based on the empirical transition model that correlates transition point with local momentum thickness (Mayle, ${ }^{53}$ Praisner and Clark, ${ }^{54}$ Roberts and Yaras $^{55}$ ). The flow pattern is in agreement with the experimental observation. In Fig. 17 we also compare the streamwise normalized velocity contours near the airfoil. A long separation bubble presents over the upper surface. A close examination reveals that flow separates at $x / c=0.2$, transition occurs at $x / c=0.54$, and reattachment happens at $x / c=0.63$, where $c$ is the airfoil chord length. These match well with measurement shown in Table 1.
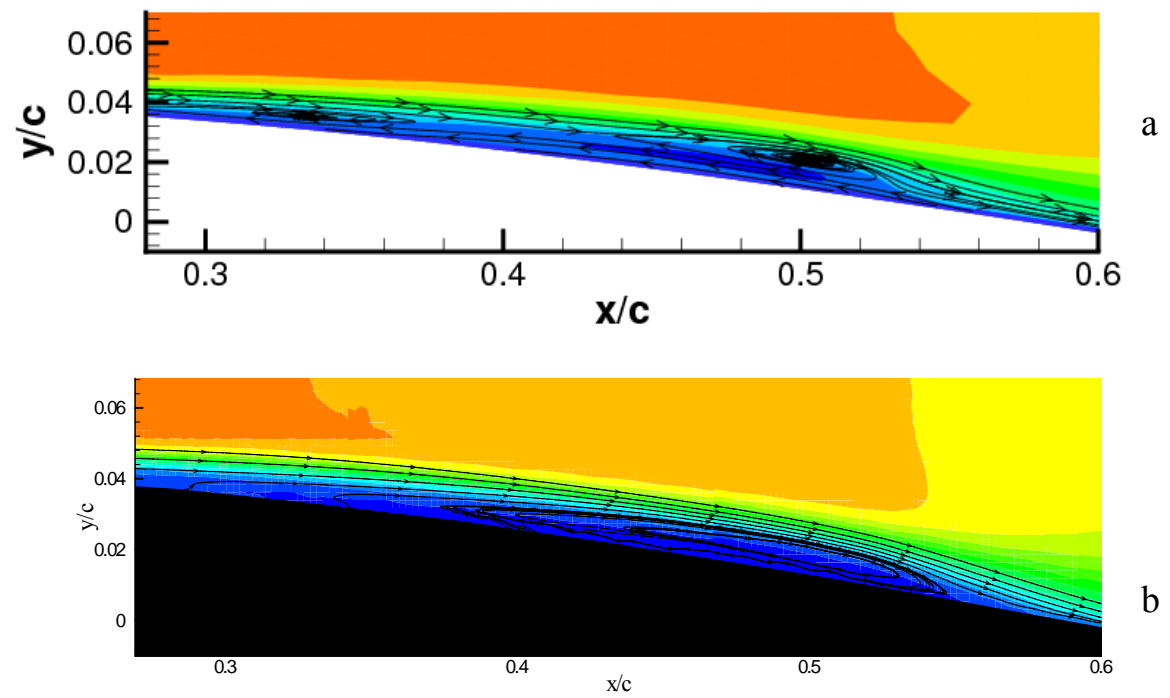

Figure 16. Streamlines over SD7003 airfoil at $4^{\circ}$ angle of attack. The Reynolds number is 60,000 . (a) Wilcox's k- $\omega$ model augmented with empirical transition model, (b) Experimental measurement of Ol et al. ${ }^{52}$ 

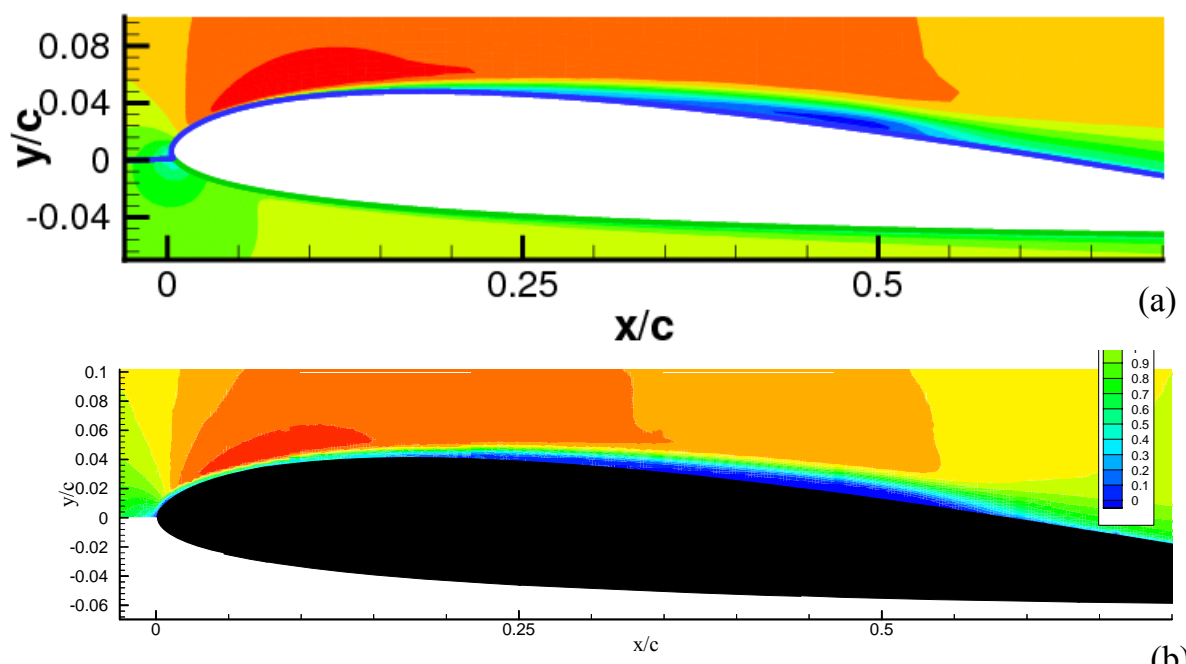

(b)

Figure 17. Normalized streamwise velocity at $4^{\circ}$ angle of attack. The Reynolds number is 60,000 . (a) CFD result with empirical transition model, (b) Experimental measurement of $\mathrm{Ol}$ et al. ${ }^{52}$

The prediction of boundary layer transition remains one of the most challenging problems in fluid mechanics. The existing transition prediction methods ranges from simple empirical relationships through those based on parallel and linear stability, such as the $\mathrm{e}^{N}$ method. ${ }^{56-58}$ The $\mathrm{e}^{N}$ methods are based on the linear stability analysis and boundary layer theory. It solves the Orr-Sommerfeld equation to evaluate the local growth rate of unstable waves based on the velocity and temperature profiles over the body. The boundary-layer profiles can be obtained by either solving the inviscid Euler equation or the Navier-Stokes equations. Transition occurs when the amplification of unstable wave reaches certain value. The $\mathrm{e}^{N}$ method remains one of the most popular methods for practical applications. The $\mathrm{e}^{N}$ method is based on the following assumptions: 1) the velocity and temperature profiles are essentially two-dimensional and steady, 2) the initial disturbance is infinitesimal, and 3) the boundary layer is thin.

One should note that MAVs frequently operate in a gusty environment, which induces high intensity of free stream unsteadiness, which can significantly affect the transition process. Second, the propeller-induced swirling flow significantly influences the transition process. Third, there exist strong spanwise flows associated with the low aspect ratio wing (Fig. 18). Fourth, when a flexible wing is used, as observed in the experiment and confirmed in numerical simulation, the wing flutters at frequencies of $O(100) \mathrm{Hz}$ (Fig. 19), and the interaction between the wing and its surrounding air modifies the flow pattern. All these factors make it difficult to employ simple transition models.
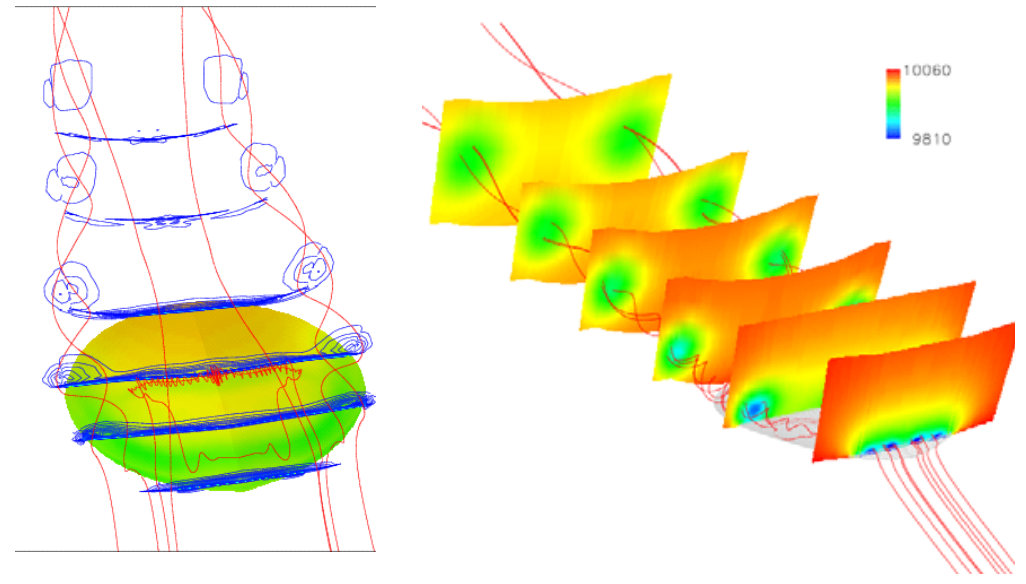

Figure 18. Streamlines, tip vortices, and pressure distributions around a rigid wing at angle of attack of $39^{\circ}$ at different cross sections. ${ }^{8}$ 
As discussed by Dick and Steelant ${ }^{59}$, the current transition models encounter difficulties even for flows over a flat plate. For low Reynolds number flows, Wilcox devised a k- $\omega$ model to predict transition. ${ }^{60}$ One of his objectives is to match the minimum critical Reynolds number, beyond which the TS wave begins forming in the Blasius boundary layer. We have tested his model on a SD7003 airfoil at a chord Reynolds number of 60,000 . In our test case the transition is caused by flow separation and flow reattaches to the surface at an angle of attack of $4^{\circ}$. Wilcox's model fails to predict the reattachment. One reason, we speculate, is that the separation introduced transition occurs before the minimum Reynolds number. Steelant and Dick, ${ }^{61}$ Suzen and Huang, ${ }^{62}$ and Suzen et al. ${ }^{63}$ incorporated the concept of intermittency factor to model the transitional flows. This can be achieved either by using conditionedaveraged Navier-Stokes equations or by multiplying the eddy viscosity by the intermittency factor. In all the cases the intermittency factor is solved from a transport equation, which is based on empirical correlations. More importantly, the onset of transition is determined by empirical correlations.

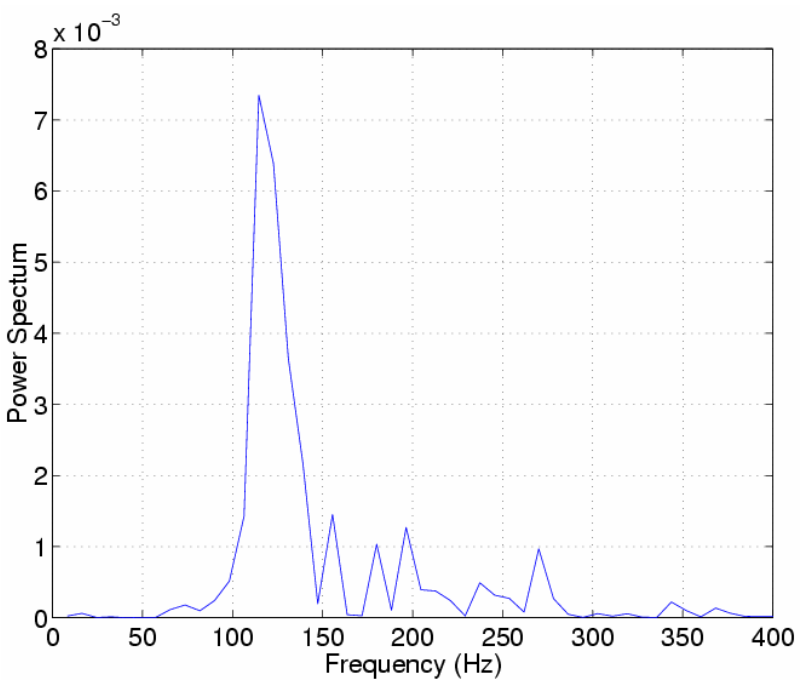

Figure 18. Flexible wing shows high frequency vibration. The numerical simulation by Lian and Shyy ${ }^{8}$ suggests a frequency around $120 \mathrm{~Hz}$.

In addition to the Reynolds-averaged

models, large eddy simulation (LES) ${ }^{64}$ and direct numerical simulation (DNS) are also being attempted. For example, Yang and Voke ${ }^{65}$ investigated boundary layer separation and transition employing LES. Yuan et al. ${ }^{66}$ studied transition over a low-Reynolds number airfoil using LES. A main difficulty in predicting the transitional flow using LES is the artificial triggering of transition by a pointwise input of turbulence energy. More efforts are clearly needed.

\section{Conclusions}

We have reviewed recent efforts made in understanding the low Reynolds number fluid dynamics related to both fixed wing and flapping wing. For flapping wing study, we have emphasized the unsteady mechanisms, including Weis-Fogh's clap-and-fling mechanism, the Reynolds number effect on the leading edge vortex, and aerodynamic peaks associated with instantaneous pitching-up and wake-capturing.

By investigating the flow structures at three Reynolds numbers, namely, 6000, 120 and 10, it is found that the structures of the leading-edge vortices vary significantly as the Reynolds number changes. At Reynolds number of 6000 , the leading edge vortex exhibits an intense, conical structure, with a sustained spanwise flow at the vortex core, breaking down at approximately three-quarters of the span towards the tip. At Reynolds number of 120, the vortex break-down disappears, and the leading-edge vortices are found to be connected to the tip vortices. Further reducing the Reynolds number to 10, a vortex ring connecting the leading edge vortex, the tip vortex and the trailing vortex is observed. Their flow structure shows more of a cylindrical than a conical form.

The hovering flight of a fruit fly shows two aerodynamic force peaks in each flapping stroke. It seems that the first peak is due to rapid vorticity increase as the wing experiences fast pitching-up rotation, while the second peak is likely to be associated with wake-capturing. Overall, these two peaks account for a large portion of the total lift.

For fixed wings, our efforts in fluid-structure interactions and laminar-turbulent transition are highlighted. As the vehicle size is reduced and dimension shrunk, the effect of free stream unsteadiness such as wind gust becomes more important for fixed wing vehicles. In different ways, the unsteady aerodynamics is crucial in advancing our understanding and design capability for both flapping and fixed wing air vehicles.

There is growing cross fertilization between computational simulation and experimental investigation. Obviously, the interactions among wing kinematics, Reynolds number, and detailed geometry are very complicated and case dependent. They need to be clarified so that we can understand the flapping wing aerodynamics, and to offer comprehensive explanations of the underlining physical mechanisms for different species as well as design concepts. 


\section{Acknowledgement}

The present work was partially sponsored by the US Air Force.

\section{Appendix}

Table 2. Experimental robotic and mechanic devices for insect flapping wing study

\begin{tabular}{|c|c|c|}
\hline Author, Year & $\begin{array}{l}\text { Wing model based } \\
\text { on: }\end{array}$ & Main features \\
\hline Bennett $(1970)^{67}$ & $\begin{array}{l}\text { Beetle } \\
\text { (Melolontha } \\
\text { vulgaris) }\end{array}$ & $\begin{array}{l}\text { - 3-D mechanical model } \\
\text { - flow visualization } \\
\text { - only wing incidence can modified } \\
\text { - do not allow force measurements }\end{array}$ \\
\hline $\begin{array}{l}\text { Spedding and } \\
\text { Maxworthy }(1986)^{68}\end{array}$ & Generic & $\begin{array}{l}\text { - 2-D mechanical model for fling mechanism } \\
\text { - only wing rotation allowed } \\
\text { - allow measurements of the aerodynamic forces using } \\
\text { a force transducer }\end{array}$ \\
\hline $\begin{array}{l}\text { Saharon and Luttgers } \\
(1987)^{69} \\
\text { Savage et al. (1979) }\end{array}$ & Dragonfly & $\begin{array}{l}\text { - 3-D mechanical model } \\
\text { - flow visualization }\end{array}$ \\
\hline $\begin{array}{l}\text { Ellington et al. } \\
(1996)^{30}\end{array}$ & Hawkmoth & $\begin{array}{l}\text { - 3-D robotic model } \\
\text { - good flow visualization (model wing }=\sim 10 \text { time the } \\
\text { insect wing size) } \\
\text { - computer-controlled wing kinematic } \\
\text { - do not allow force measurements }\end{array}$ \\
\hline $\begin{array}{l}\text { Dickinson et al. } \\
(1999)^{29}\end{array}$ & Fruit fly & $\begin{array}{l}\text { - 3-D robotic model } \\
\text { - PIV technology (scaled-up wing) } \\
\text { - computer-controlled wing kinematic } \\
\text { - allow measurements of shear forces using a 2-D force } \\
\text { transducer }\end{array}$ \\
\hline $\begin{array}{l}\text { Lehmann }(2004)^{37} \\
\text { Maybury et al. }(2005)^{71}\end{array}$ & $\begin{array}{l}\text { Fruit-fly and dragon } \\
\text { fly }\end{array}$ & $\begin{array}{l}\text { - 3-D robotic model } \\
\text { - DPIV technology (scaled-up wing) } \\
\text { - computer-controlled wing kinematic } \\
\text { - allow measurements of shear forces using a 6-D force } \\
\text { transducer }\end{array}$ \\
\hline
\end{tabular}


Table 3. Principal numerical studies of insect flapping flight

\begin{tabular}{|l|l|l|l|}
\hline Author, Year & Fluid governing equations & Method & Moving boundary tracking \\
\hline Smith (1996) & 3-D potential flow & Panel method & $\begin{array}{l}\text { Time depended boundary } \\
\text { conditions }\end{array}$ \\
\hline $\begin{array}{l}\text { Liu and Kawachi } \\
(1998)^{38}\end{array}$ & $\begin{array}{l}\text { 3-D Navier-Stokes in body- } \\
\text { fitted coordinates }\end{array}$ & $\begin{array}{l}\text { Artificial } \\
\text { compressibility }\end{array}$ & $\begin{array}{l}\text { Grid re-meshing. } \\
\text { Analytical based on the initial } \\
\text { grid and wing kinematics }\end{array}$ \\
\hline Wang et al. (2004) & $\begin{array}{l}\text { 2-D Navier-Stokes for vorticity } \\
\text { in elliptic coordinates. }\end{array}$ & Finite difference & $\begin{array}{l}\text { Time depending boundary } \\
\text { conditions }\end{array}$ \\
\hline Mittal et al. (2002) & 2-D Navier-Stokes equations & Finite volume & $\begin{array}{l}\text { Immersed Boundary Method } \\
\text { on fixed Cartesian grid }\end{array}$ \\
\hline $\begin{array}{l}\text { Ramamurti and } \\
\text { Sandberg }(2002)^{42}\end{array}$ & $\begin{array}{l}\text { 3-D Navier-Stokes in ALE } \\
\text { formulation } \\
\text { (Arbitrary Lagrangian Eulerian) }\end{array}$ & Finite Element & $\begin{array}{l}\text { Grid re-meshing } \\
\text { Spring analogy and smoothing } \\
\text { on unstructured grid }\end{array}$ \\
\hline $\begin{array}{l}\text { Sun and Tang } \\
(2002)^{31,32}\end{array}$ & $\begin{array}{l}\text { 3-D Navier-Stokes in body- } \\
\text { fitted coordinates. }\end{array}$ & $\begin{array}{l}\text { Artificial } \\
\text { compressibility }\end{array}$ & $\begin{array}{l}\text { Time dependent coordinate } \\
\text { transformation }\end{array}$ \\
\hline $\begin{array}{l}\text { Sun and Lan } \\
(2004)^{50}\end{array}$ & $\begin{array}{l}\text { 3-D Navier-Stokes in body- } \\
\text { fitted coordinates. }\end{array}$ & $\begin{array}{l}\text { Artificial } \\
\text { compressibility }\end{array}$ & Overset moving grid \\
\hline $\begin{array}{l}\text { Gilmanov and } \\
\text { Sotiropoulos(2005) }\end{array}$ & 3-D Navier-Stokes & Finite difference & $\begin{array}{l}\text { Immersed Boundary Method } \\
\text { on fixed Cartesian grid }\end{array}$ \\
\hline
\end{tabular}

\section{References}

${ }^{1}$ Pornsin-Sirirak, T. N., Lee, S. W., Nassef, H., Grasmeyer, J., Tai, Y. C., Ho, C. M., and Keennon, M., "MEMS Wing Technology for a Battery-powered Ornithopter," The Thirteenth Annual International Conference on Micro Electro Mechanical Systems, Miyazaki, Japan, Jan. 23-27, 2000, pp. 799-804.

${ }^{2}$ Grasmeyer, J. M., and Keennon, M. T., "Development of the Black Widow Micro Air Vehicle," AIAA Paper 2001-0127, presented at the 39th Aerospace Sciences Meeting and Exhibit, Reno, NV, Jan. 8-11, 2001.

${ }^{3}$ Ifju, P. G., Jenkins, A. D., Ettingers, S., Lian, Y., and Shyy, W., "Flexible-Wing-Based Micro Air Vehicles," AIAA Paper 2002-0705, presented at the 40th Aerospace Sciences Meeting and Exhibit, Reno, NV, Jan. 14-17, 2002.

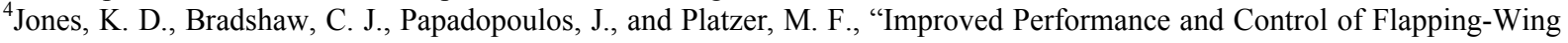
Propelled Micro Air Vehicles," AIAA Paper 2004-0399, presented at the 42nd Aerospace Sciences Meeting and Exhibit, Reno, NV, Jan. 5-8, 2004.

${ }^{5}$ Jones, K. D., and Platzer, M. F., "Experimental Investigation of the Aerodynamic Characteristics of Flapping-Wing Micro Air Vehicles," AIAA Paper 2003-0418, presented at the 41st Aerospace Sciences Meeting and Exhibit, Reno, NV, Jan. 6-9, 2003.

${ }^{6}$ Shyy, W., Berg, M., and Ljungqvist, D., "Flapping and Flexible Wings for Biological and Micro Air Vehicles," Progress in Aerospace Sciences, Vol. 35, July 1999, pp. 455-506.

${ }^{7}$ Waszak, R. M., Jenkins, N. L., and Ifju, P., "Stability and Control Properties of an Aeroelastic Fixed Wing Micro Aerial Vehicle," AIAA Paper 2001-4005, presented at the AIAA Atmospheric Flight Mechanics Conference and Exhibit, Montreal, Canada, Aug. 6-9, 2001.

${ }^{8}$ Lian, Y., and Shyy, W., "Numerical Simulations of Membrane Wing Aerodynamics for Micro Air Vehicle Applications," Journal of Aircraft, Vol. 42, No. 4, 2005, pp. 865-873.

${ }^{9}$ Ho, S., Nassef, H., Pornsinsirirak, Tai, Y-C., and Ho, C-M., "Unsteady Aerodynamics and Flow Control for Flapping Wing Flyers," Progress in Aerospace Sciences, Vol. 39, 2003, pp. 635-681.

${ }^{10}$ Mueller, T. J., and DeLaurier, J. D., “Aerodynamics of Small Vehicles,” Annual Review of Fluid Mechanics, Vol. 35, Jan. 2003, pp. 89-111.

${ }^{11}$ Lian, Y., Shyy, W., Viieru, D., and Zhang, B. N., "Membrane Wing Aerodynamics for Micro Air Vehicles," Progress in Aerospace Sciences, Vol. 39, 2003, pp. 425-465.

${ }^{12}$ Shyy, W., Ifju, P.G. and Viieru, D., "Membrane Wing-Based Micro Air Vehicles," Applied Mechanics Reviews, Vol. 58, July, 2005, pp. 283-301.

${ }^{13}$ Heathcote, S., Martin, D., Gursul, I., "Flexible Flapping Airfoil Propulsion at Zero Freestream Velocity,"AIAA Journal, Vol. 42, No. 11, 2004, pp. 2196-2204. 
${ }^{14}$ Tuncer I. H., and Platzer M. F., “Thrust Generation Due to Airfoil Flapping,” AIAA Journal, Vol. 34, No. 2, 1996, pp. 324331.

${ }^{15}$ Gursul I., "Review of Unsteady Vortex Flows over Slender Delta Wings," Journal of Aircraft, Vol. 42, No.2, 2005, pp. 299-319.

${ }^{16}$ Gursul I., "Vortex Flows on UAVs: Issues and challenges," Aeronautical Journal, Vol. 108, No. 1090, 2004, pp.597-610.

${ }^{17}$ Visbal, M. R., Gordnier, R. E., "Numerical Simulation of the Interaction of a Transitional Boundary Layer with a 2-D Flexible Panel in the Subsonic Regime," Journal of Fluids and Structures, Vol. 19, No.7, 2004, pp. 881-903.

${ }^{18}$ Gordnier, R. E., Visbal, M. R., "High-fidelity Computational Simulation of Nonlinear Fluid-structure Interactions," Aeronautical Journal, Vol. 109, No. 1097, 2005, pp. 301-312.

${ }^{19}$ Choudhuri, P. G., Knight, D., D., Visbal, M. R., "2-Dimensional Unsteady Leading-Edge Separation on a Pitching Airfoil," AIAA Journal, Vol. 32, No. 4, pp. 673-681.

${ }^{20}$ Visbal, M. R., Gaitonde, D. V., "On the Use of Higher-order Finite-difference Schemes on Curvilinear and Deforming Meshes," Journal of Computational Physics, Vol. 181, No. 1, 2002, pp. 155-185.

${ }^{21}$ Sarkar, S., Venkatraman, K., "Numerical Simulation of Thrust Generating Flow Past a Pitching Airfoil," Computers and Fluids, Vol. 35, No. 1, 2006, pp. 16-42.

${ }^{22}$ Chen, X. Y., Zha, G. C., "Fully Coupled Fluid-structural Interactions Using an Efficient High Resolution Upwind Scheme," Journal of Fluids and Structure, Vol. 20, No. 8, 2005, pp. 1105-1125.

${ }^{23}$ Okong'o, N., Knight, D. D., "Implicit Unstructured Navier-Stokes Simulation of Leading Edge Separation over a Pitching Airfoil," Applied Numerical Mathematics, Vol. 27, No. 3, 1998, pp. 269-308.

${ }^{24}$ Ellington, C. P., "The Aerodynamics of Hovering Insect Flight. I. The Quasi-steady Analysis," Philosophical Transactions of the Royal Society of London, Series B, Vol. 305, Feb. 1984, pp. 1-15.

${ }^{25}$ Azuma, A., The Biokinetics of Flying and Swimming, Springer-Verlag, Tokyo, 1992, pp. 25-29.

${ }^{26}$ Pennycuick, C. J., Bird Flight Performance: A Practical Calculation Manual, Oxford: Oxford University Press, 1989.

${ }^{27}$ Tennekes, H., The Simple Science of Flight, The MIT Press, Cambridge, MA, 1996.

${ }^{28}$ Dickinson, M. H., and Götz, K. G., "Unsteady Aerodynamic Performance of Model Wings at Low Reynolds Numbers," Journal of Experimental Biology, Vol. 174, Jan. 1993, pp. 45-64.

${ }^{29}$ Dickinson, M. H., Lehmann, F-O., Sane, S., "Wing Rotation and the Aerodynamic Basis of Insect Flight," Science, Vol. 284, Jun. 1999, pp. 1954-1960.

${ }^{30}$ Ellington, C. P., Berg, C. van den, Willmott, A. P., and Thomas, A. L. R., "Leading-edge Vortices in Insect Flight," Nature, Vol. 384, Dec. 1996, pp. 626-630.

${ }^{31}$ Sun, M., and Tang, J., "Unsteady Aerodynamic Force Generation by a Model Fruit Fly Wing in Flapping Motion," Journal of Experimental Biology, Vol. 205, Jan. 2002, pp. 55-70.

${ }^{32}$ Sun, M., and Tang, J., "Lift and Power Requirements of Hovering Flight in Drosophila virilis," Journal of Experimental Biology, Vol. 205, Aug. 2002, pp. 2413-2427.

${ }^{33}$ Weis-Fogh, T., "Quick Estimate of Flight Fitness in Hovering Animals, Including Novel Mechanisms for Lift Production," Journal of Experimental Biology, Vol. 59, 1973, pp. 169-230.

${ }^{34}$ Lighthill, M. J., "On the Weis-Fogh Mechanism of Lift Generation,” Journal of Fluid Mechanics, Vol. 60, 1973, pp. 1-17.

${ }^{35}$ Maxworthy, T., "Experiments on the Weis-Fogh Mechanism of Lift Generation by Insects in Hovering Flight. Part 1. Dynamics of the 'fling'," Journal of Fluid Mechanics, Vol. 93, 1979, pp. 47-63.

${ }^{36}$ Lehmann, F.-O., Sane, S. P., and Dickinson, M., "The Aerodynamic Effects of Wing-wing Interaction in Flapping Insect Wings," Journal of Experimental Biology, Vol. 208, Aug. 2005, pp. 3075-3092.

${ }^{37}$ Lehmann, F-O., "The Mechanisms of Lift Enhancement in Insect Flight," Naturwissenschaften, Vol. 91, March 2004, pp. 101-122.

${ }^{38}$ Liu, H., and Kawachi, K., “A Numerical Study of Insect Flight,” Journal of Computational Physics, Vol. 146, Oct. 1998, pp. 124-156.

${ }^{39}$ Berg van den, C., and Ellington, C. P., “The Three-dimensional Leading-edge Vortex of a 'Hovering' Model Hawkmoth. Philosophical Transactions of the Royal Society of London, Series B, Vol. 352, 1997, pp. 329-340.

${ }^{40}$ Sane S. P., and Dickison M. H., "The Aerodynamic Effects of Wing Rotation and a Revised Quasi-steady Model of Flapping Flight”, Journal of Experimental Biology, Vol. 205, Apr. 2002, pp. 1087-1096.

${ }^{41}$ Birch, J. M., and Dickinson, M. H., "Spanwise Flow and the Attachment of the Leading-edge Vortex," Nature, Vol. 412, No. 6848, 2001, pp. 729-733.

${ }^{42}$ Ramamurti, R., and Sandberg, W., “A Three-dimensional Computational Study of the Aerodynamic Mechanisms of Insect Flight," Journal of Experimental Biology, Vol. 205, May 2002, pp. 1507-1518.

${ }^{43}$ Sane, S. P., "The Aerodynamics of Insect Flight," Journal of Experimental Biology, Vol. 206, Dec. 2003, pp. $4191-4208$.

${ }^{44}$ Walker, J. A., "Rotational Lift: Something Different or More of the Same?" Journal of Experimental Biology, Vol. 205, September 2002, pp. 3783-3792.

${ }^{45}$ Wang, Z. J., Birch, J. M., and Dickinson, M. H., "Unsteady Forces and Flows in Low Reynolds Number Hovering Flight: Two-dimensional Computations vs Robotic Wing Experiments," Journal of Experimental Biology, Vol. 207, Jan. 2004, pp. 449460.

${ }^{46}$ Shyy, W., Computational Modeling for Fluid Flow and Interfacial Transport, Elsevier, the Netherlands, 1994.

${ }^{47}$ Tang, J., "High Lift Mechanism and Power Consumption in Hovering of Fruit Fly Drosophila virilus", Ph.D. Dissertation, Beijing University of Aeronautics and Astronautics, P. R. China, 2001. 
${ }^{48}$ Wu, J. H., and Sun, M., "Unsteady Aerodynamic Forces of a Flapping Wing,” Journal of Experimental Biology, Vol. 207, January 2004, pp. 1137-1150.

${ }^{49}$ Sun, M., and Du, G., "Lift and Power Requirements of Hovering Insect Flight," Acta Mechanica Sinica, Vol. 19, 2003, pp. 24-31.

${ }^{50}$ Sun, M., and Lan. S., "A Computational Study of the Aerodynamic Forces and Power Requirements of Dragonfly (Aeschna juncea) Hovering,” Journal of Experimental Biology, Vol. 207, May 2004, pp. 1887-1901.

${ }^{51}$ Birch, J. M., and Dickinson, M. H., "The Influence of Wing-wake Interaction on the Production of the Aerodynamic Forces in Flapping Flight," Journal of Experimental Biology, Vol. 206, Jul. 2003, pp. 2257-2272.

${ }^{52}$ Ol, M., McAuliffe, B. R., Hanff, E. S., Scholz, U., and Kaehler, C., "Comparison of Laminar Separation Bubble Measurements on a Low Reynolds Number Airfoil in Three Facilities," AIAA Paper 2005-5149, 23rd AIAA Applied Aerodynamics Conference, Toronto, Jun. 6-9, 2005.

${ }^{53}$ Mayle, R. E., "The Role of Laminar-Turbulent Transition in Gas Turbine Engine," Journal of Turbomachinery, Vol. 113, No. 4, 1991, pp. 509-537.

${ }^{54}$ Praisner, T. J., and Clark, J. P., "Predicting Transition in Turbomachinery, Part I-A Review and New Model Development," ASME Paper No. GT2004-54108, 2004.

${ }^{55}$ Roberts, S. K., and Yaras, M. I., "Effects of Surface Roughness Geometry on Separation Bubble Transition," ASME Paper No. GT2005-68664, 2005.

${ }^{56}$ Smith, A. M. O., and Gamberoni, N., “Transition, Pressure gradient, and Stability Theory,” Douglas Aircraft Report ES26388, 1956.

${ }^{57}$ Van Ingen, J. L., “A Suggested Semi-Empirical Method for the Calculation of the Boundary Layer Transition Region,” Inst. Of Technogy, Department of Aeronautics and Engineering, Report VTH-74, Delft, Holland, 1956.

${ }^{58}$ Drela, M., and Giles, M. B., "Viscous-Inviscid Analysis of Transonic and Low Reynolds Number Airfoils," AIAA Journal, Vol. 23, No. 10, 1987, pp. 1347-1355.

${ }^{59}$ Dick, E., and Steelant, J., "Coupled Solution of the Steady Compressible Navier-Stokes Equations and the k- $\varepsilon$ Turbulence Equations with a Multigrid Method," Applied Numerical Mathematics, Vol. 23, Feb. 1997, pp. 49-61.

${ }^{60}$ Wilcox, D., Comparison of Two-Equation Turbulence Models for Boundary Layers with Pressure Gradient, AIAA Journal, Vol. 30, No. 8, 1993, pp. 1414-1421.

${ }^{61}$ Steelant, J., and Dick, E., "Modeling of Bypass Transition with Conditioned Navier-Stokes Equations Coupled to an Intermittency Transport Equation," International Journal of Numerical Methods in Fluids, Vol. 23, Aug. 1996, pp. 193-220.

${ }^{62}$ Suzen, Y. B., and Huang, P. G., "Modeling of Flow Transition Using an Intermittency Transport Equation," Journal of Fluids Engineering, Vol. 122, Jun. 2000, pp. 273-284.

${ }^{63}$ Suzen, Y. B., Xiong, G., and Huang, P. G., "Predictions of Transitional Flows in a Low-Pressure Turbine Using an Intermittency Transport Equation," AIAA Journal, Vol. 40, No. 2, 2002, pp. 254-266.

${ }^{64}$ Strelets, M., "Detached Eddy Simulation of Massively Separated Flows," AIAA Paper 2001-0879, presented at the 39th Aerospace Sciences Meeting and Exhibit, Reno, NV, Jan. 8-11, 2001.

${ }^{65}$ Yang, Z., and Voke, P., "Large Eddy Simulation of Boundary-Layer Separation and Transition at a Change of Surface Curvature,” Journal of Fluid Mechanics Vol. 439, Jul. 2001, pp. 305-333.

${ }^{66}$ Yuan, W., Khalid, M., Windte, J., Scholz, U., and Radespiel, R., “An Investigation of Low-Reynolds-Number Flows Past Airfoils," AIAA Paper 2005-4607, 23rd AIAA Applied Aerodynamics Conference, Toronto, Jun. 6-9, 2005.

${ }^{67}$ Bennett, L., "Insect Flight: Lift and Rate of Change of Incidence," Science, Vol. 167, 1970, pp. 177-179.

${ }^{68}$ Spedding, G. R., Maxworthy, T., "The Generation of Circulation and Lift in a Rigid Two-dimensional Fling," Journal of Fluid Mechanics, Vol. 165, 1986, pp. 247-272.

${ }^{69}$ Saharon, D., Luttges, M. W., "Three-dimensional Flow Produced by a Pitching-plunging Model Dragonfly Wing," 25th AIAA Aerospace Sciences Meeting, AIAA Paper 1987-0121, Reno, NV, Jan. 1987.

${ }^{70}$ Savage, S. B., Newman B. G., and Wong, D. T.-M., "The Role of Vortices and Unsteady Effects during the Hovering Flight of Dragonflies," Journal of Experimental Biology, Vol. 83, 1979, pp. 59-77.

${ }^{71}$ Maybury, W. J., and Lehmann, F.-O., "The Fluid Dynamics of Flight Control by Kinematic Phase Lag Variation between Two Robotic Insect Wings," Journal of Experimental Biology, Vol. 207, No. 26, Dec. 2004, pp. 4707-4726.

${ }^{72}$ Smith, M. J. C., "Simulating Moth Wing Aerodynamics - Towards the Development of Flapping-wing Technology," AIAA Journal, Vol. 34, No. 7, 1996, pp. 1348-1355.

${ }^{73}$ Mittal, R., Utturkar, Y., and Udaykumar, H.S., "Computational Modeling and Analysis of Biomimetic Flight Mechanisms," AIAA Paper 2002-865, presented at 40th AIAA Aerospace Sciences Meeting and Exhibit, Reno, NV, Jan. 2002.

${ }^{74}$ Gilmanov, A., and Sotiropoulos, F., "A Hybrid Cartesian/immersed Boundary Method for Simulating Flows with 3D, Geometrically Complex, Moving Bodies,” Journal of Computational Physics, Vol. 207, No. 2, 2005, pp. 457-492. 